PROCEEDINGS OF THE

AMERICAN MATHEMATICAL SOCIETY

Volume 133, Number 3, Pages 805-807

S 0002-9939(04)07683-X

Article electronically published on September 20, 2004

\title{
UNE REMARQUE SUR LA SYMÉTRIE ASYMPTOTIQUE DE LA FONCTION DE GREEN
}

\author{
HAMID-REZA FANAÏ
}

(Communicated by Michael Handel)

\begin{abstract}
Dans cette note, nous montrons que la symétrie asymptotique de la fonction de Green dans le cas des variétés compactes de courbure négative implique que la variété est localement symétrique.
\end{abstract}

Soit $(M, g)$ une variété riemannienne compacte de courbure sectionnelle strictement négative. Une mesure invariante par le flot géodésique définie sur $S M$ le fibré unitaire tangent est la mesure harmonique $\nu$. On peut la représenter à partir de la famille de mesures harmoniques $\left\{\nu_{x}\right\}_{x \in \widetilde{M}}$ sur $\partial \widetilde{M}$ le bord géométrique du revêtement universel. On sait que pour $x, y \in \widetilde{M}$, les deux mesures harmoniques $\nu_{x}$ et $\nu_{y}$ sont équivalentes et on a

$$
\frac{d \nu_{y}}{d \nu_{x}}(\xi)=K(x, y, \xi)
$$

où $K(x, y, \xi)$ est le noyau de Poisson. Il est défini à partir de la fonction de Green $G(x, y)$ dont l'existence est assurée par $\left[\mathrm{AS}\right.$. On a en fait: $K(x, y, \xi)=\lim _{z \rightarrow \xi} \frac{G(y, z)}{G(x, z)}$ et l'existence de cette limite est une conséquence du principe de Harnack. Ceci permet de définir pour tout vecteur $v \in S \widetilde{M}, \tau(v)=\left.\frac{d}{d t}\right|_{t=0} \ln K(v(0), v(t), v(+\infty))$, où $v(t)$ désigne la géodésique définie par $v$, et on sait que $\tau(v)$ est invariante par l'action du groupe fondamental $\pi_{1}(M)$ et descend donc sur $S M$. On suppose que $\omega^{s}$ (resp. $\omega^{u}$ ) est la mesure harmonique sur $S M$ associée au feuilletage stable (resp. instable) $(\underline{Y})$.

Dans $[\mathbf{F}$, nous avons considéré l'invariance par symétrie des mesures harmoniques $\nu_{x}$ et la symétrie asymptotique de la fonction de Green. Rappelons que la fonction de Green est dite asymptotiquement symétrique si pour tout $x \in \widetilde{M}$ et tout $\xi \in \partial \widetilde{M}$, on a

$$
\lim _{y \rightarrow \xi} \frac{G(x, y)}{G\left(x,-{ }_{x} y\right)}=1
$$

où $y$ varie sur la géodésique définie par le couple $(x, \xi)$ et $-_{x} y$ désigne l'image symétrique de $y$ par rapport à $x$ sur cette géodésique. Nous avons montré dans ce

Received by the editors October 31, 2003.

2000 Mathematics Subject Classification. Primary 37D40, 53D25.

Key words and phrases. Flot géodésique, mesures invariantes.

L'auteur remercie V. Kaimanovich pour son aide. Le soutien du Conseil de Recherches de l'Université Technologique Sharif est également remercié.

(C)2004 American Mathematical Society Reverts to public domain 28 years from publication 
cas, que pour tout vecteur $v$, on a $([\mathrm{F}]$, p. 164),

$$
K(v(0), v(t), v(+\infty))=K(v(0), v(t), v(-\infty)) \cdot \exp \left(2 h_{\text {top }} t\right)
$$

où $h_{\text {top }}$ est l'entropie topologique du flot géodésique. En fait pour $v=(x, \xi) \in S \widetilde{M}$, si $y=v(t)$ on a par la symétrie asymptotique de $G(x, y)$,

$$
K(x, y, \xi)=K\left(x, y,-_{x} \xi\right) \cdot \lim _{R \rightarrow-\infty} \frac{G(v(0), v(R))}{G(v(0), v(R-2 t))}
$$

et on voie que cette dernière limite ne change pas lorsque $v$ bouge par le flot géodésique. Ceci implique alors que $\tau(v)+\tau(-v)=2 h_{\text {top }}$.

La quantité suivante, introduite par V. Kaimanovich $([\underline{\mathrm{K}})$

$$
\alpha=\int_{S M} \tau(v) d \omega^{s}(v)
$$

vérifie $\alpha \leq h_{\nu}$, où $h_{\nu}$ désigne l'entropie du flot géodésique par rapport à $\nu$. Il est bien connu que l'on a égalité si et seulement si $(M, g)$ est asymptotiquement harmonique $([\underline{\mathrm{K}}]$ ), et donc localement symétrique grâce au résultat de rigidité de Besson, Courtois et Gallot.

Maintenant, notons que l'idée d'utiliser le feuilletage stable dans la définition de $\alpha$ n'est pas une obstruction, et en travaillant avec le feuilletage instable, on peut démontrer de la même manière que

$$
\int_{S M} \tau(v) d \omega^{u}(v) \leq h_{\nu}
$$

car la mesure harmonique $\nu$ est invariante par la symétrie (flip-invariant).

Maintenant nous pouvons conclure en intégrant l'égalité $\tau(v)+\tau(-v)=2 h_{\text {top }}$ par rapport à $\omega^{s}$. On a

$$
\int_{S M} \tau(v) d \omega^{s}(v)+\int_{S M} \tau(-v) d \omega^{s}(v)=2 h_{\mathrm{top}},
$$

et puisque

$$
\int_{S M} \tau(-v) d \omega^{s}(v)=\int_{S M} \tau(v) d \omega^{u}(v)
$$

ceci implique

$$
\int_{S M} \tau(v) d \omega^{u}(v) \geq h_{\mathrm{top}}
$$

On trouve alors $\alpha=h_{\nu}$. On termine donc la preuve du résultat suivant.

Théorème 1. Soit $(M, g)$ une variété riemannienne compacte de courbure strictement négative. Si la fonction de Green est asymptotiquement symétrique, alors $(M, g)$ est localement symétrique.

Remarque. Ce théorème est comparable au cas où la variété est supposée d'être harmonique. Dans ce cas, on sait que la fonction de Green $G(x, y)$ ne dépend que de $d(x, y)$ et elle est donc asymptotiquement symétrique. 


\section{REFERENCES}

[AS] M. T. Anderson et R. Schoen, Positive harmonic functions on complete manifolds of negative curvature. Ann. Math., 121 (1985), 429-461. MR.0794369 (87a:58151)

[F] H.-R. Fanaï, Sur quelques problèmes liés au flot géodésique. Bull. Belg. Math. Soc., 10 (2003), 161-167. MF 2015195

[K] V. A. Kaimanovich, Brownian motion and harmonic functions on covering manifolds. An entropy approach. Soviet Math. Dokl., 33 (1986), 812-816. MR0852647 (88k:58163)

[Y] C. B. Yue, Brownian motion on Anosov foliations and manifolds of negative curvature. J. Diff. Geometry, 41 (1995), 159-183. MR.1316554 (95k:58123)

Department of Mathematical Sciences, Sharif University of Technology, P.O. Box 11365-9415, TEHRAN, Iran

E-mail address: fanai@sharif.ac.ir 